\title{
Current Research, Challenges, and Perspectives of Biotechnology: An Overview
}

\author{
Nguyen Duc Bach ${ }^{1}$ and Ly Thi Bich Thuy ${ }^{2}$ \\ ${ }^{1}$ Faculty of Biotechnology, Vietnam National University of Agriculture, Hanoi 131000, \\ Vietnam; \\ ${ }^{2}$ Institute of Biotechnology, Vietnam Academy of Science and Technology, Hanoi \\ 123200, Vietnam
}

\begin{abstract}
Biotechnology is defined as biology-based technology using organisms or their parts to make or modify products or to improve characteristics of plants, animals, and microorganisms for the demands of human beings. Biotechnology profoundly impacts various fields such as agriculture, animal husbandry and veterinary, industry, food science, pharmaceutics and medicine, environment, fine chemistry, biofuels, forensics, and nanotechnology. Nowadays, biotechnology and bioindustries are becoming integral parts of the knowledge-based economy, and therefore, biotechnology has become a powerful and indispensable tool for the development of all countries in the world. As a rule, biotechnology also requires regulatory policies to control genetically modified organisms and derived products to avoid risks to biodiversity, human health, the environment, and ethical issues. This article reviews the current research, challenges, and perspectives of biotechnology as an integration of both life sciences and applied sciences.
\end{abstract}

\section{Keywords}

Animal biotechnology, Plant biotechnology, Environmental biotechnology, Microbial technology, Medicinal biotechnology.

\section{Introduction}

Biotechnology is a broad field of the life sciences and applied sciences that is defined as any technological application using biological systems, living organisms, or derivatives thereof, to make or modify products or processes for the demands of humans (Chekol and Gebreyohannes, 2018; Jayne et al., 2002). Biotechnology can be also seen as multiple disciplines of basic biological sciences and engineering such as molecular biology, biochemistry, cell biology, embryology, genetics, microbiology, bioinformatics, genetic engineering, biomedical engineering, food technology, nanobiotechnology, and bio-manufacturing, etc. (Chekol and Gebreyohannes, 2018). In addition, biotechnology also provides 


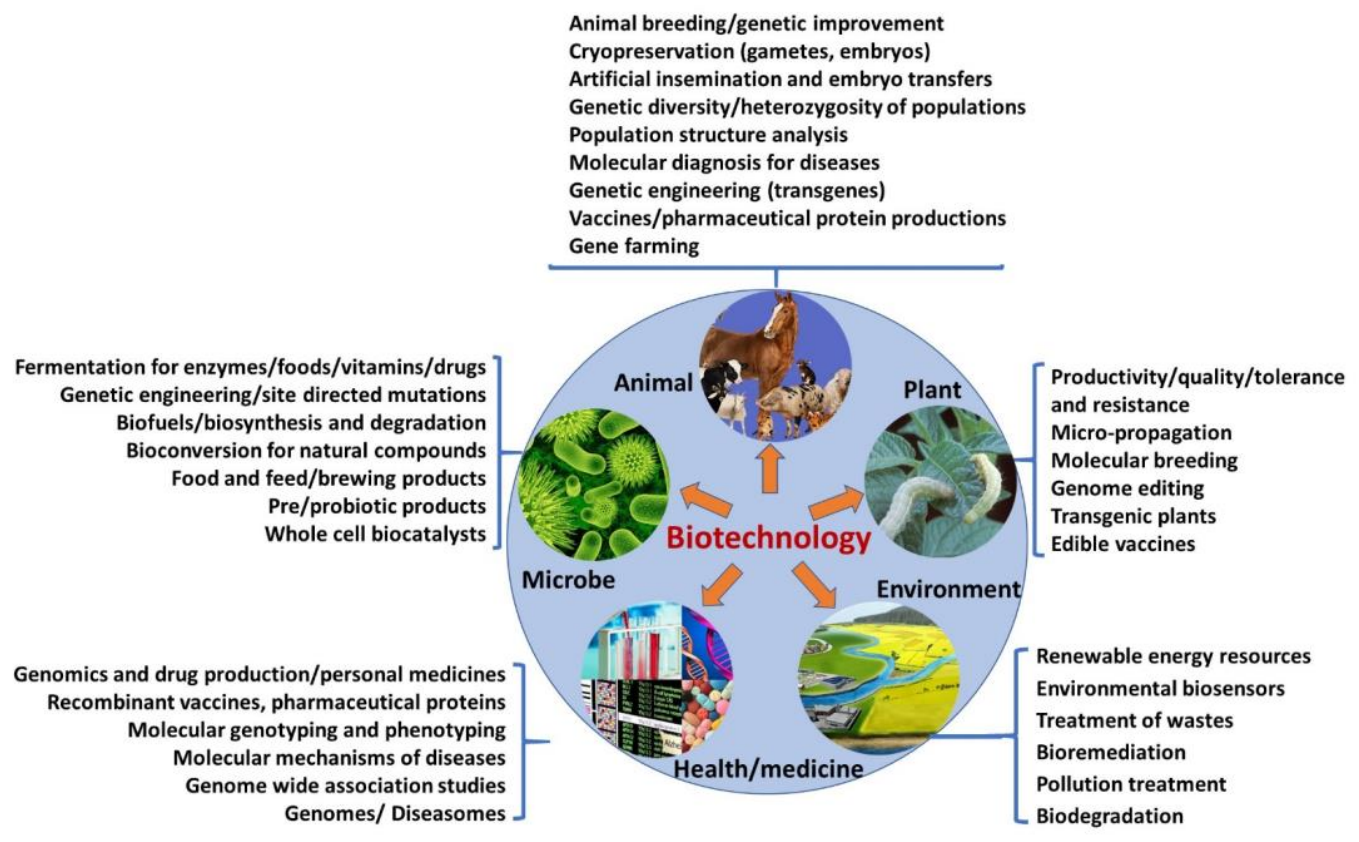

Figure 1. Major areas of biotechnology

methods and powerful tools to support the basic research of many other related fields (Jungbauer et al., 2012). Although biotechnology involves a wide range of areas, in this review article, the five major areas of biotechnology are focused on, namely plant biotechnology, microbial biotechnology, animal biotechnology, environmental biotechnology, and health biotechnology (Figure 1).

\section{Current Research in Broad Areas of Biotechnology}

\section{Plant biotechnology}

In the area of plant biotechnology, plant breeding takes a center role in the creation, selection, and improvement of crop varieties to fulfil the never-ending requests by farmers and consumers. For years, micro-propagation has taken an important role in in vitro vegetative propagation of plants by tissue culture. Micropropagation has several advantages over conventional propagation methods including preservation of genotype constitution, rapid multiplication of shoot and roots, preparation of virus-free materials, and easier transportation and storage. Cultures of apical meristems, induction of axillary and adventitious shoots, and regeneration by somatic embryogenesis and organogenesis are common techniques in micropropagation (Atanassova and Keiper, 2018; Singh et al., 2018).

In plant breeding, the ultimate aims are to improve yields, increase the quality or profitable value, and develop resistance against pests or unfavorable conditions. Of the currently used techniques, marker-assisted backcrossing is the most common approach using molecular markers to assist in the selection of new desired varieties. The principle involves incorporating a gene of interest into an elite variety that is already well adapted. So far, many agronomic traits such as high yield, disease resistance, biotic and abiotic stresses tolerance, food quality, and fragrance have been introduced into many types of crop varieties through markerassisted backcrossing. To date, crops such as soybean, corn, canola, and cottonseed oil have been genetically engineered to be resistant against pathogens and herbicides, to have better nutrient profiles, or to tolerate unfavorable conditions (Bawa and Anilakumar, 2013). A typical example of plant biotechnology is the use of a toxic protein $(\mathrm{Bt})$ from Bacillus thuringiensis to control insects in corn production (Fleming et al., 2018). 
Advanced techniques in genome sequencing, especially next-generation sequencing (NGS) and bioinformatic tools, provide huge databases for identifying and understanding the functions of genes. Recently, available information from highly polymorphic DNA markers such as single nucleotide polymorphisms (SNPs) or microsatellites have been very useful for plant breeding, and functional and evolutionary studies that have made plant breeding become more precise and less time-consuming (Perez-de-Castro et al., 2012; Mammadov et al., 2018).

Genetic engineering can be used to modify the amino acid composition of plant proteins to increase the nutritional value of staple crops. Crops in development include soybeans with modified fatty acid profiles and higher essential amino acids content (Ufaz and Galili, 2008); increased sensory qualities in tomatoes and fruits (flavor, aroma, and texture) (Klee, 2010); golden rice which has extra iron and vitamin A (Dubock, 2017); potatoes with more nutritionally available starch and with improved amino acid content (Bagri et al., 2018); crops which produce beta-carotene; oilseed rape containing a special type of polyunsaturated fatty acid (PUFA); soybean containing predominantly cis-monounsaturated fatty acid (oleic acid) (Hefferon, 2015); peppers and melons with improved flavor are currently in field trials; edible vaccines such as a cholera vaccine in potatoes; improved tomatoes with delayed softening; and reduced levels of toxicants (cyanogenic glycosides in cassava and mycotoxins in cereal fermentations), allergic reactions, or anti-nutritional factors (phytates) allowing a wider range of plants to be used as food crops (Bawa and Anilakumar, 2013; Maryam et al., 2017a).

Next-generation genome sequencing (NGS) technology enables the sequencing of whole genomes or transcriptomes. SNPs are new targets for tagging and linkage analysis. More recently, the development of genome editing technologies such as transcription activator-like effector nuclease (TALEN), zinc finger nuclease (ZFN), and clustered regularly interspaced short palindromic repeats (CRISPR/Cas9) have triggered the dawn of genome editing (Ju et al.,
2018). As a trend, any change in the genome, including a specific DNA sequence or indels, can be made with unprecedented precision and specificity (Bhat et al., 2017).

\section{Animal biotechnology}

Animal biotechnology provides new avenues for genetic improvement in the production of farm animals such as promoting growth, increasing nutrient intake efficiency, increasing growth rates, enhancing milk production and nutrition quality, reducing environmental impacts, and improving disease resistance, reproductive performance, fecundity, hair, and fiber (Wheeler, 2013) (Figure 2). Cryopreservation of gametes or gene banking is a promising technique in biotechnology for long-term preservation and storage of sperm or eggs (Alexandrov et al., 2013; Škrbić, 2018). The technology of cryopreservation of fish spermatozoa has been adopted for animal husbandry (Asturiano et al., 2017).

For years, artificial insemination and embryo transfers have been two important techniques used in animal breeding. Artificial insemination technology supports improving quality, monitoring gender, minimizing the transmission of venereal diseases, reducing the number of breeding males, and controlling the pedigree record. Embryo transfer aims to improve genetic merit, increase the number of offspring, and minimize diseases (Wheeler, 2013; Murray and Maga, 2016).

In animal breeding, the genetic uniqueness of populations is measured by the relative genetic distances of such populations from each other. DNA polymorphisms are a reliable source of information for the estimation of genetic distances. Although restriction fragment length polymorphisms (RFLP) and randomly amplified polymorphic DNA (RAPD) are simple techniques, they are very effective to estimate the genetic uniqueness of populations and molecular structure of the population. In addition, microsatellite and minisatellite sequences have been used in the DNA fingerprinting technique to evaluate genetic diversity, polymorphic information content, and heterozygosity of a population (Yadav et al., 2017). 


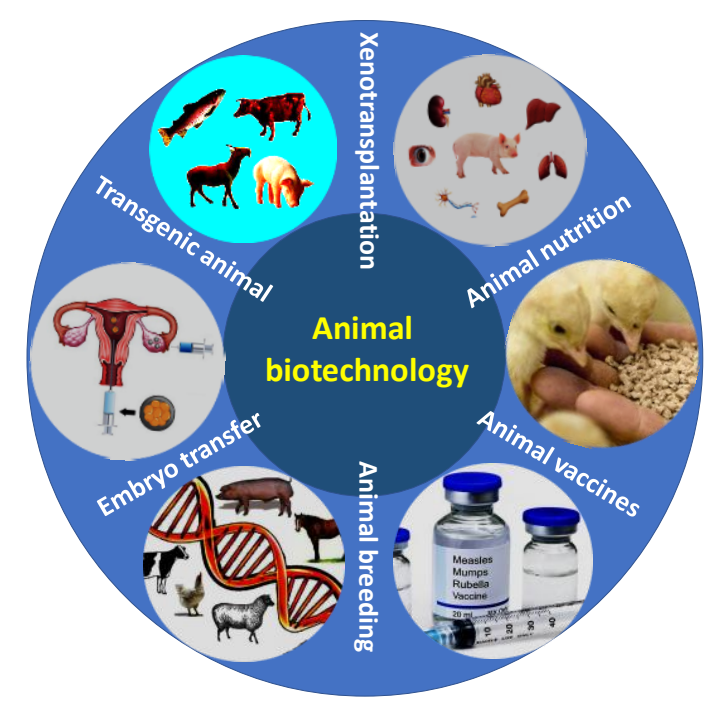

Figure 2. Major areas of animal biotechnology

Molecular diagnosis has been proven to be a powerful and accurate tool for the identification and control of animal diseases. The advances of biotechnological techniques facilitate the detection and characterization of pathogens in infected hosts. Monoclonal antibodies are used to detect specific parasite antigens by a simple ELISA technique. The advent of PCR has considerably enhanced the sensitivity of DNA detection tests to identify infectious diseases. Other diagnostic techniques such as nucleic acid hybridization and restriction endonuclease mapping can be applied to distinguish infections caused by bacteria or viruses even in cases in which symptoms are clinically identical or the infection cannot be distinguished antigenically with available serological reagents (Yang et al., 2013; Yadav et al., 2017; Škrbić, 2018).

Advances in biotechnology, especially recombinant DNA technology, have improved the production of effective vaccines and tools for the diagnosis of infectious diseases. The process of vaccine development has increased at all levels, from the investigation of immune responses to the production and delivery of protective antigens to target species. As a result, the production of recombinant pathogen proteins (antigens) and serological tests have enabled the development of various types of vaccines over a short period of time (McCullers and Dunn, 2008; Nascimento and Leite, 2012).

\section{Microbial biotechnology}

For years, fermentation has been widely applied in the production of microbial cultures, enzymes, flavors, fragrances, food additives, and a range of other high value-added products. By using both traditional and molecular approaches, bacteria, yeasts, and molds have been used widely in the food, dairy, and brewing industries. In order to obtain desired microbial strains for fermentation, traditional methods of genetic improvement such as classical mutagenesis and conjugation in combination with high-throughput selection have been applied (Maryam et al., 2017b). In recent years, recombinant DNA technology has been used to modify the genetic material of bacteria, yeasts, and molds. In addition, through protein engineering, novel enzymes with modified structures for thermal stability, substrate specificity, or the ability to work under extreme conditions have been developed (Li et al., 2012; Gurung et al., 2013). Directed evolution is one of the main methods currently used for protein engineering in microbes (Adrio and Demain, 2014). Many strains of microbes have been genetically modified to increase enzyme production, or for substrate specificity or stereoselectivity. For example, chymosin in the stomach of calves has been successfully produced by DNA technology in Kluyveromyces lactis, Escherichia coli, and Aspergillus niger var. awamori for cheese production. 
Glucoamylase found in yeasts is produced by biotechnology and is added in feedstock for better utilization of carbohydrates, or is used in the beverage industry for increasing alcohol production or producing low-calorie beer (Ogata et al., 2017).

In recent years, probiotics are probably one of the most important research topics in both food and feed. Probiotic products have been successfully used in aquaculture to enhance both the internal and external microbial environment, and the current trend is to replace antibiotics by probiotics for ecological considerations (Omole, 2017).

Metabolic engineering is an important tool for industrial biotechnology by redirecting precursor metabolic fluxes based on the manipulation of enzymes, transport systems, and regulatory functions in the cell. Metabolic engineering is also applied to produce large amounts of valuable metabolites or natural secondary compounds that are difficult to extract from their natural sources, or infeasible via chemical synthesis. One example is the production of the amino acid lysine from the genetically modified microorganism Corynebacterium glutamicum (Leuchtenberger, 2005). Likewise, a variety of important tools including protein engineering, metabolic engineering, synthetic biology, systems biology, and downstream processing have been applied for the production of antibiotics, vaccines, vitamins, enzymes, and useful products (Tang and Zhao, 2009). As examples, valencene, a sesquiterpene originally found in the peel of Valencia oranges, and nootkatone in grapefruit peel are now produced by microbial fermentation (Kutyna and Borneman, 2018). Libraries of the mutants generated by directed evolution, rational design, and high throughput screening assisted by modern techniques such as fluorescence labelling, flow cytometry, and microfluidic arrays have been developed to identify interesting mutants containing enzymes with desirable properties (Tang and Zhao, 2009).

\section{Health and medicinal biotechnology}

In the areas of health and medicine, modern biotechnology is a promising tool for both research and application. Understanding the molecular mechanism of diseases or disorders in the aspect of pharmacogenomics is very useful for genetic testing, gene therapy, and drug production. Gene therapy is an example of using DNA as a pharmaceutical agent to treat diseases. In germ line gene therapy, germ cells are modified by the introduction of functional genes, which are integrated into their genomes to replace a mutated or defected gene.

The success of the Human Genome Project (HGP) brought a huge opportunity for discovering the underlying structures and functions of genes in the human genome. The 1000 Genomes Project provides by far the most detailed catalogue of human genetic variation. The obtained data from this project are valuable tools for many fields of genetics, medicine, pharmacology, biochemistry, and bioinformatics that support the understanding of the mechanisms of genetic diseases.

Pharmaceutical drugs can be produced by chemically modified molecules derived from biological sources. For instance, recombinant human insulin first became commercially manufactured in 1982. Recombinant human growth hormone (hGH) has greatly improved the long-term treatment of children lacking hGH. For haemophilia patients, factors VIII and IX involved blood coagulation can be produced by cloning and the over-expression of respective genes in CHO cells (Singh et al., 2016a). For years, antibiotics, amino acids, enzymes, enzyme inhibitors, nucleotides, vitamins, organic acids, vaccines, and polysaccharides with applications in the field of medicine to improve human health have been produced by extraction technology or chemical synthesis. However, the production of these products is now being switched toward industrial biotechnology-based processes.

Recently, biopharmaceuticals from plants and microorganisms, particularly bacteria, fungi, yeast, and microalgae, can be produced using fermentation processes or direct extraction from plant biomass by transgenic technology or metabolic regulation. Many bioactive compounds from actinomycetes, myxobacteria, eubacteria, algae, and fungi are reported to be 
produced at large scales in Saccharomyces cerevisiae or Escherichia coli (Ramana et al., 2017). For example, paclitaxel (Taxol) is a plant-derived natural isoprenoid product able to inhibit cancer cells. Paclitaxel was originally isolated from the Pacific yew tree but is now commercially produced through biosynthetic strategies (Li et al., 2015).

\section{Environment biotechnology}

Environmental biotechnology is a discipline involving the application of biological systems and processes in waste treatment and management. The use of biological-based processes to remediate environmental pollutants is known as bioremediation. The term "bioremediation" has been used to describe the process of using microorganisms to degrade or remove hazardous components of wastes from the environment (Glazer and Nikaido, 1995). Biodegradation is defined as a natural process whereas bioremediation has been developed as a way to stimulate or accelerate the degradation of pollutants and, therefore, render a site free from contamination. Bioremediation applies biological processes and biotechnical methods, and enzyme bioreactors are being developed that will pretreat some industrial wastes and food waste components and allow their removal through the sewage system rather than through solid waste disposal mechanisms (Dua et al., 2002). Microorganisms are used as whole cell biocatalysts for processes such as bioleaching, biodetergent, biotreatment of pulp, biotreatment of wastes (bioremediation), biofiltration, aquaculture treatments, biotreatment of textiles, biocatalysts, biomass fuel production, and biomonitoring.

Biotransformation of organic contaminants in the natural environment has been extensively studied to understand microbial ecology, physiology, and evolution for their potential in bioremediation. Molecular techniques can be used to increase the level of a particular protein, enzyme, or series of enzymes in bacteria with the goal of increasing the reaction rate. The easiest way to create an appropriate genetically engineered strain is to begin with an organism that already possesses much of the necessary degradative enzymatic machinery.
Advances in genetic and protein engineering techniques enable tailor-made genetically engineered microorganisms to work as "designer biocatalysts". Through the genetic engineering of metabolic pathways, it is possible to extend the range of substrates that an organism can utilize. So far, many modified microbes are able to degrade harmful chemical wastes such as ethylbenzene, trichloroethylene, toluene, chlorobenzene, 3,4-dichloro-1-butene and indole, radionuclides, chlorobenzene, nitrotoluene, and organic solvents. Biotechnology techniques such as DNA shuffling, random priming, or staggered extension processes can accelerate the recombination rate or assembly of existing genetic material as a kind of molecular evolution. These techniques, therefore, can be selected to guide the evolution of enzymes or pathways in specialized directions. The introduction of an exogenous plasmid carrying foreign genes for conversion or degradation is an effective way to modify native microorganisms. Catabolic enzymes can be engineered for the enhancement of degradation rates or to broaden substrate specificity.

The development of DNA probes based on aptamer technology can be used for the very sensitive detection of toxic or waste products based on specific molecular interactions. Highthroughput screening of DNA oligos that specifically bind with heavy metals or harmful factors can be applied to develop environmental biosensors (Nguyen et al., 2017).

\section{Current Challenges and Future Perspectives of Biotechnology}

\section{Plant biotechnology}

In the last decades, a large number of agricultural species have been partly or completely sequenced. With a rapid accumulation of biological sequences, comparisons of entire genomes or specific regions of interest reveal precious information about the properties and functions of various genes. With the advances of NGS technologies in both reducing costs and increasing performance rates, and "omics" technologies, agricultural researchers can bridge the gap in the 
relationship between genotype-phenotype and environmental factors (Ohashi et al., 2015). To a certain extent, one could state that the current phase of the Arabidopsis genome project is that it has completed its work in knowing the functions of almost all Arabidopsis genes, interactomes, phenomics, metabolic pathways, and network regulations. Therefore, it would be the right time to develop technologies to transfer this knowledge to other plants, especially crops.

As the demands of human beings continuously increases, e.g. increased nutritional value of grains, fewer or no allergens or antinutritional factors in food products, increased shelf lives of fruits and vegetables, and higher contents of vitamins and micronutrients found in cereals, new technologies, especially in transgenes and gene editing, will be required. For example, genetic engineering has been applied to modify the fatty acid profiles in soybean oil to increase the proportion of polyunsaturated fatty acid (PUFA). The development of edible vaccines by genetic engineering in plants may provide an efficient approach to increase and strengthen the performance of the immune system of animals by controlling their daily diet (Kamthan et al., 2016).

Although there are many debates over large-scale commercialization and use of genetically modified organisms (GMOs), transgenic crops, and recombinant DNA technology, these technologies are the future trends in plant biotechnology, and understanding the roles of genes governing complex traits to actively improve agronomic performance or control adaptations to abiotic stresses is a matter of concern (Maghari and Ardekani, 2011). The complex traits of interest include a crop's ability to grow efficiently in drought, salinity, acidic, or aluminiumcontaining soils, competition with weeds, flowering time, heterosis, and durable resistance to diseases. In the next decades, it would not be surprising if some of these complex traits are integrated in crop plants by genetic engineering. It should be also mentioned here that although gene/genome editing technologies have been successfully tried in many research laboratories, there exists a large gap in successfully creating new crop varieties with desired traits for human consumption. However, the use of gene editing techniques in crop plants will be the future solutions toward setting up new strategies for the sustainable development of agriculture in the situations of the world's growing population and climate changes (Maghari and Ardekani, 2011; Moshelion and Altman, 2015).

The depletion of fossil fuels leading to an increase of energy prices requires new processes for the production of renewable energy sources called biofuels. Biofuels are derived from renewable feedstocks such as ethanol from food crops, biomass, or byproducts of agricultural production. Lignocelluloses are promising materials for biofuel production through enzymatic fermentation and chemical transformations (Den et al., 2018). The modification or alteration of the properties of the polysaccharide profile in the cell walls of plant materials is a great challenge for biotechnology in the degradation of the stable polymer chain into sugar molecules for further fermentation and conversion (Popa, 2018).

\section{Animal biotechnology}

In animal reproduction, selecting the sex of embryos is still challenging. Semen sexing technology is used for producing offspring of the desired sex, either male or female. This technique relies on the principle of flow cytometric separation of fluorescence labeled sex-chromosomes. However, one of the main drawbacks of this technique is the low number of sexed sperms produced and the occurrence of sperms being damaged during the sorting process that reduces the efficiency of fertilization in later steps (Espinosa-Cervantes and Cordova-Izquierdo, 2013). Therefore, new generation flow cytometers with high sorting rates or new methods for sexed sperm separation should be developed (Asma U.l. Husna. et al., 2017).

In molecular-assisted breeding, new molecular technologies will change the traditional approach from phenotyping to genotyping. As the genomes of domesticated livestock animals including chicken, pig, cow, sheep, and horse, etc. are completely sequenced 
(Bai et al., 2012), the new dawn of the postgenomic era will be started. Entire genome research with the support of sophisticated bioinformatics tools allow large data sets to be analyzed to uncover the hidden information inside biological sequences. Breeders can take advantage of novel molecular breeding tools for animal production to ensure food security in changing environments.

Gene farming refers to the concept of using transgenic farm animals as biological factories to manufacture commercially valuable products in their milk. Many genes for growth hormones have been constructed with engineered promoters to produce human pharmaceutical peptides and proteins in the milk of mice, rabbits, sheep, goats, swine, and cattle. It is likely that this approach is feasible to produce human pharmaceutical products rather than conventional industrial procedures (Bosze and Hiripi, 2012). Although many attempts have been carried out, the first two therapeutic agents to be isolated from the milk of transgenic animals, $\mathrm{C} 1$ inhibitor and antithrombin, are now commercialized. In the near future, recombinant human proteins and monoclonal antibodies could be produced using transgenic animals and become available for practical use (Maksimenko et al., 2013).

Cloning technology would help cattle producers reproduce genetically valuable seedstock animals. This technology increases the accuracy of selection in the tested breeding herds. However, the problem is that cloned animals often suffer from severe injuries or are not able to reproduce. With this scenario, stem cell or somatic cell technologies should be developed to overcome drawbacks in current cloning methodologies in order to increase efficient and economically feasible reproduction for cattle producers (Kim et al., 2012). In animal biotechnology, the issues of animal welfare should also be taken into consideration. Depending on one's personal beliefs, some people oppose the use of animals for any purpose, while others have specific concerns about the impacts that genetic engineering and cloning may bring by producing human therapeutic or industrial proteins (Nabavizadeh, 2016).
In the area of transplantation of living cells, tissues, or organs, there is always a shortage of organs for clinical implantation in patients who need a replacement organ at the end-stage of failure. Xenotransplantation is the idea of transplanting living cells, tissues, or organs from one species to another. For humans, tissues or organs from some animals from the order Primates or from pigs could be candidates for transplantation. However, the lifespans of the donor animals are shorter than humans; therefore, the aging of the grafted tissues at a quicker rate is still a challenge in xenotransplantation technology (Hryhorowicz, 2017). In addition, similarly to the protest against animal testing, animal rights activists have also objected to xenotransplantation on ethical grounds. Therefore, only a few temporarily successful cases of xenotransplantation have thus far been published.

\section{Microbial biotechnology}

Microbial biotechnology is integrated with many different practical areas including (i) agricultural practices, (ii) microbial enzymes for industry, and (iii) environment treatments (Figure 3). In the future, research in microbial biotechnology will still be focused on these 3 main areas in various application fields. The screening of new strains of bacteria, fungi, and microalgae for production of high value-added products via fermentation processes or degradation of toxic compounds in the soil and water or for production of new industrial enzymes are the important missions in microbial biotechnology (Matassa et al., 2016).

Recently, the production of biofertilizers, biopesticides, bioherbicides, and bioinsecticides has become a new trend in the sustainable development of agriculture. Microbial inoculants, also known as soil inoculants, are agricultural amendments that use beneficial endophytes (microbes) to promote plant health (Singh and Strong, 2016). Many of the microbes involved form symbiotic relationships with the target crops where both parties mutually benefit (Chandler et al., 2011). While microbial inoculants are applied to improve plant nutrition, they can also be used to promote plant growth by stimulating plant hormone production. 


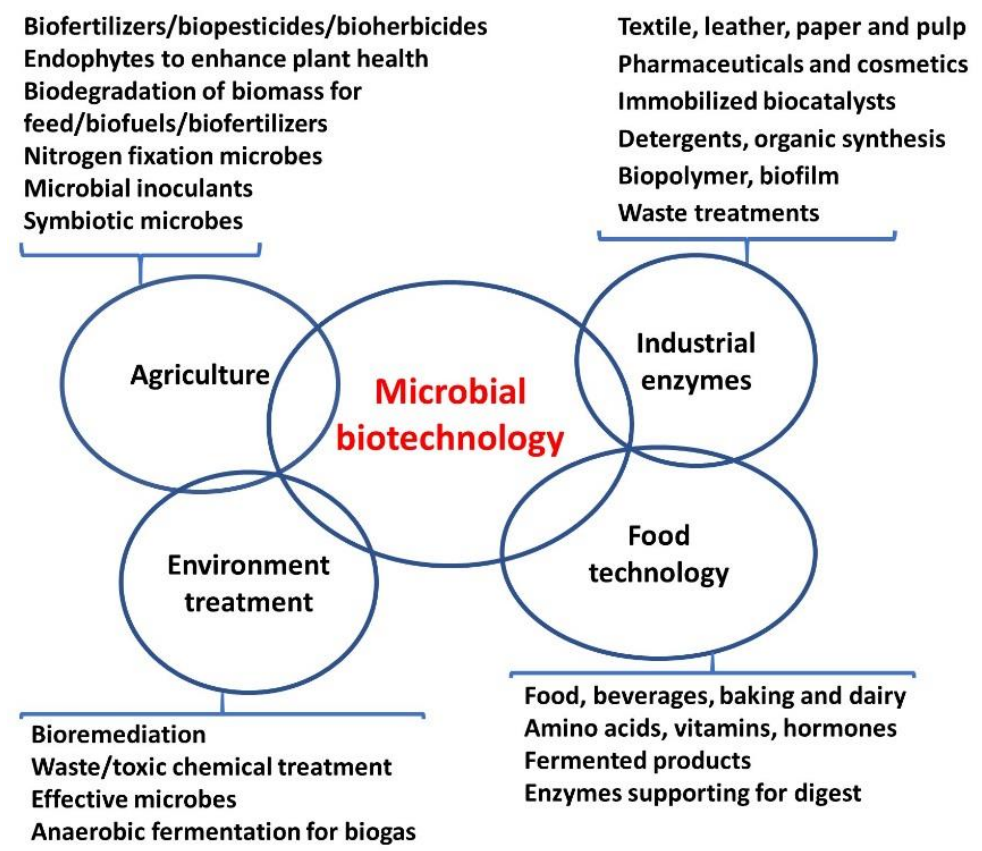

Figure 3. Focused areas of microbial biotechnology

Due attention is needed regarding Azotobacter, Acetobacter, Trichoderma, Bacillus thuringiensis, and Azospirillum and their application in various cereal and vegetable crops. These biofertilizers should be integrated with organic manures and chemical fertilizers to enhance the soil organic carbon and maintain sustainability in field and horticultural crops (Gopalakrishnan et al., 2015). Byproducts in agriculture and forestation production such as stubble, straw, and sawdust contain stubborn polymers (lignin, cellulose, and hemicellulose) and are a challenge for the development of new technology for biodegradation to convert them into biofuels, feeds, and biofertilizers (Kilbane, 2016).

Industrial enzymes are commercially used in a variety of industries such as textiles, leather, paper and pulp, biopolymers, food and feed, beverages, cosmetics, detergents, organic synthesis, waste management, pharmaceuticals, baking, and dairy. These areas require a wide range of industrial enzymes, and commonly used enzymes are palatase, lipozyme, lipase, cellulase, amylase, xylose isomerase, resinase, penicillin amidase, amidase, asparaginase, bromelain, urokinase, subtilisin, xylanases, and $\beta$-lactamase (Gurung et al., 2013; Singh et al., 2016b). Although reactions in organisms are efficiently performed by enzymes under physiological conditions, industrial conditions are far different with high substrate concentrations, sheering forces, high or low temperatures, and organic solvents. In addition, the requirements of regiospecific, chemospecific, and estereospecific reactions are challenging for industrial and pharmaceutical enzymes (Chapman et al., 2018). Therefore, most enzymes found in soil and water microbes are not able to display their desired activities under industrial conditions. Therefore, enzymes with desired activities under industrial conditions could be obtained by optimizing the newer technology process conditions and by protein engineering using directed evolution (Baweja et al., 2016).

In addition, immobilized biocatalysts can also offer the possibility of wider and more economical exploitation of biocatalysts in industry, waste treatment, medicine, and in the development of bioprocess monitoring devices like the biosensor (Mohamad et al., 2015). Microbial enzymes can degrade toxic or harmful chemical compounds from the wastes of industrial production and domestic chemicals such as phenolic compounds, nitriles, and amines by enzymatic degradation or conversion (Singh et al., 2016b; Karigar and Rao, 2011). 


\section{Health and medicinal biotechnology}

The completion of the human genome project and the recent 1000 Genomes Project give a great opportunity for researchers to convert the DNA sequence data from many different genotypes into useful information (Devuyst, 2015). Although the advent of NGS and genome assembly have rapidly changed biotechnology, functional genomics is still a big challenge in gene identification, analysis of gene interactions, and the relationships between genotypes and phenotypes in complex diseases. In addition, underlying the network of the diseasome is necessary to understanding genedisease interactions (Carter et al., 2013).

Research in genomics and proteomics are seen as the next important supply sources of innovative future drug design targets or personalized medicine. By taking advantage of scientific breakthroughs, state-of-the-art "omics" technologies such as genomics, proteomics, transcriptomics, glycomics, metabolomics, pharmacogenomics, and toxicogenomics, and systems biology, these powerful health and medicinal biotechnology tools would become unprecedented in understanding diseases and developing new drugs (Dunisławska et al., 2017).

Recombinant DNA technologies will be intensively applied in the production of a wide range of drugs, hormones, and enzymes, including vaccines against the influenza virus, treatments for rheumatoid arthritis, and prevention of blood coagulation, malaria, cholera, herpes, rheumatoid arthritis, tuberculosis, cancer, and gastrointestinal diseases. In the future, challenging problems such as HIV, cancers, asthma, Parkinson's disease, and Alzheimer's disease will hopefully be controlled by effective drugs. Various groups of biopharmaceuticals including antibiotics, blood factors, hormones, growth factors, cytokines, enzymes, vaccines, and monoclonal antibodies are expected to be developed.

\section{Environmental biotechnology}

Environmental challenges require newer technologies for environmental control, protection, and remediation. Many approaches continue to exploit the potential of beneficial microorganisms and plants for sustainable development. Although effective microorganisms (EM) have been used widely in various products for environmental treatment and management, there is still a need for more efficient products because the components of wastes and disposal are becoming more complicated (Vujic et al., 2015).

Enzyme engineering is used to improve biodegradation in order to reuse treated wastewater. At present, new technologies are being applied for soil remediation and the cleanup of contaminated sites such as those contaminated with organic chemicals (dioxin, toluene, chlorobenzene, and organic solvents) (Das and Chandran, 2011; Nzila et al., 2016). The implementation of anaerobic digestion to treat biowaste as an alternative and renewable energy resource for fossil fuels is emerging worldwide. As a must, future developments should be sustainable in such a way to develop clean processes and products with less harmful and reduced environmental impacts.

Finally, the use of genetically modified organisms in industrial processes could be considered a biohazard to the environment. The balance between environmental and economic benefits needs to be solved to reduce environmental impacts and enhance sustainability by biotechnology (Coelho and Garcia Diez, 2015). In a global view, especially in agriculture, the intricate balances between hosts, pests, humans, and the environment should be seen as a challenge for biotechnology in the future.

\section{Conclusions}

In recent decades, biotechnology has been shown to be a new powerful tool that has profoundly impacted many areas of the life sciences and application fields in agriculture, animal husbandry and veterinary, industry, health and medicines, and environment. Moreover, the development of biotechnology by itself also promotes the progress of fundamental and applied research in other areas of the natural sciences. As a result, biotechnology is seen as a pivotal element in the $4^{\text {th }}$ industrial and agriculture revolution. At 
present, biotechnology is emerging as an indispensable tool for the development of social economics and environment protection in all countries of the world.

\section{Acknowledgements}

This study is funded by the grant 03/FIRST/1.a/VNUA from the Ministry of Science and Technology under the framework of the World Bank projects.

\section{References}

Adrio J. L. and Demain A. L. (2014). Microbial enzymes: tools for biotechnological processes. Biomolecules. Vol 4 (1). pp. 117-139.

Alexandrov T. Chernyavsky I., Becker M., Eggeling F. V. and Nikolenko S. (2013). Analysis and interpretation of imaging mass spectrometry data by clustering mass-to-charge images according to their spatial similarity. Analytical Chemistry. Vol 85 (23). pp. 11189-11195.

Asma U.l. Husna., Awan M. A., Mehmood A., Sultana T., Shahzad Q., Ansari M. S., Rakha B. A., Saqlan Naqvi S. M. and Akhter S. (2017). Sperm sexing in Nili-Ravi buffalo through modified swim up: Validation using $\mathrm{SYBR}^{\circledR}$ green real-time PCR. Animal Reproduction Science. Vol 182. pp. 69-76.

Asturiano J. F., Cabrita E. and Horvath A. (2017). Progress, challenges and perspectives on fish gamete cryopreservation: A mini-review. General Comparative Endocrinology. Vol 245. pp. 69-76.

Atanassova A. and Keiper F. (2018). Plant breeding innovation: A global regulatory perspective. Cereal Chemistry. Vol 95 (1). pp. 8-16.

Bagri D. S., Upadhyay D. C., Jain S. K. and Upadhyay C. P. (2018). Biotechnological improvement of nutritional and therapeutic value of cultivated potato. Frontiers in Bioscience (Scholar Edition). Vol 10. pp. 217-228.

Bai Y., Sartor M. and Cavalcoli J. (2012). Current status and future perspectives for sequencing livestock genomes. Journal of Animal Science Biotechnology. Vol 3 (1). Article 8.

Bawa A. S. and Anilakumar K. R. (2013). Genetically modified foods: safety, risks and public concerns-a review. Journal of Food Science and Technology. Vol 50 (6). pp. 1035-1046.

Baweja M., Nain L., Kawarabayasi Y. and Shukla P. (2016). Current Technological Improvements in Enzymes toward Their Biotechnological Applications. Frontiers in Microbiology. Vol 7 (965). Article 965, 13 pages. doi: 10.3389/fmicb.2016. 00965.
Bhat S. A., Malik A. A., Ahmad S. M., Shah R. A., Ganai N. A., Shafi S. S. and Shabir N. (2017). Advances in genome editing for improved animal breeding: A review. Veterinary World. Vol 10 (11). pp. 1361-1366.

Bosze Z. and Hiripi L. (2012). Recombinant protein expression in milk of livestock species. Methods Mol Biol. Vol 824. pp. 629-641.

Carter H., Hofree M. and Ideker T. (2013). Genotype to phenotype via network analysis. Current Opinion in Genetics \& Development. Vol 23 (6). pp. 611-621.

Chandler D., Bailey A. S., Tatchell G. M., Davidson G., Greaves J. and Grant W. P. (2011). The development, regulation and use of biopesticides for integrated pest management. Philosophical transactions of the Royal Society of London. Series B, Biological Sciences. Vol 366 (1573). pp. 1987-1998.

Chapman J., Ismail A. E. and Dinu C. Z. (2018). Industrial Applications of Enzymes: Recent Advances, Techniques, and Outlooks. Catalysts 2018. Vol 8 (6). Article 238, 26 pages. doi:10.3390/catal8060238.

Chekol C. and Gebreyohannes M. (2018). Application and Current Trends of Biotechnology: a Brief Review. Austin Journal of Biotechnology \& Bioengineering. Vol 5 (1). pp. 1-8.

Coelho A. C. and Garcia Diez J. (2015). Biological Risks and Laboratory-Acquired Infections: A Reality That Cannot be Ignored in Health Biotechnology. Frontiers in Bioengineering and Biotechnology. Vol 3 (56). Article 56.

Das N. and Chandran P. (2011). Microbial degradation of petroleum hydrocarbon contaminants: an overview. Biotechnology Research International. Vol 2011 (10). Article 941810, 13 pages.

Den W., Sharma V. K., Lee M, Nadadur G. and Varma R. S. (2018). Lignocellulosic Biomass Transformations via Greener Oxidative Pretreatment Processes: Access to Energy and Value-Added Chemicals. Frontiers in Chemistry. Vol 6 (141). Article 141. doi: 10.3389/fchem.2018.00141.

Devuyst O. (2015). The 1000 Genomes Project: Welcome to a New World. Peritoneal Dialysis International. Vol 35 (7). pp. 676-677.

Dua M., Singh A., Sethunathan N. and Johri A. K. (2002). Biotechnology and bioremediation: successes and limitations. Applied Microbiology and Biotechnology. Vol 59. pp. 143-152.

Dubock A. (2017). An overview of agriculture, nutrition and fortification, supplementation and biofortification: Golden Rice as an example for enhancing micronutrient intake. Agriculture \& Food Security. Vol 6. Article 59, 20 pages. doi: 10.1186/s40066-017-0135-3.

Dunisławska A., Łachmańska J., Slawinska A. and Siwek M. (2017). Next generation sequencing in animal science - A review. Animal Science Papers and Reports. Vol 35 (3). pp. 205-224. 